\title{
True Malignant Mixed Tumor of Submandibular Salivary Gland - A Case Report
}

\author{
Dr. S. Subbiah M.S., M.Ch ${ }^{1}$, Dr. Sujay Susikar M.S., M.Ch ${ }^{2}$, Dr. \\ A. N. Gurumoorthy M.Ch postgraduate ${ }^{2}$. \\ ${ }^{1,2}$ Department Of Surgical Oncology, Centre For Oncology, Government Royapettah Hospital, \\ Kilpauk Medical College, Chennai, Tamilnadu, India.
}

Abstract: Malignant mixed tumors of salivary gland are extremely rare tumors defined by the presence of malignant epithelial and mesenchymal components together. We report here a case of carcinosarcoma arising from a recurrent pleomorphic adenoma of submandibular salivary gland in a 36 year old woman. Histology showed a monomorphic picture dominated by malignant mesenchymal component with mixed nature proven by immuno histochemical analysis. We reviewed literature and discuss the theories on tumurogeneis, role of immunohistochemistry in diagnosis and clinicopathological features of this rare entity.

Keywords: Carcinosarcoma, Immunohistochemistry (IHC), Malignant mixed tumors, Submandibular salivary gland

\section{Introduction}

Malignant mixed tumors account for less than $2 \%$ of all salivary gland tumors [1]. The term encompasses three distinct pathological entities: Carcinoma ex pleomorphic adenoma, Carcinosarcoma (true malignant mixed tumor) and benign metastasizing pleomorphic adenoma [1]. The former represents malignant transformation of pre-existing longstanding or recurrent pleomorphic adenoma [2].

Malignant mixed tumour (carcinosarcoma) is an extremely rare aggressive biphasic malignant tumor with both stromal and epithelial components undergoing malignant degeneration. It accounts for $0.04-0.16 \%$ of all salivary gland tumors [1]. To date, there are only about 70 cases reported in medical literature most of which are case reports on tumors of parotid origin.

We report here a case of a malignant mixed tumor arising from submandibular gland with extensive mesenchymal component almost entirely replacing the epithelial component, malignant biphasic nature proven only by immunohistochemical study.

\section{Case report}

A 36 year old female, previously operated for right submandibular gland swelling, histopathology reported as pleomorphic adenoma, presented to us after one year later with a recurrent lesion in the submandibular region. Clinical examination showed a non tender submandibular swelling of varying consistency with restricted mobility and fixity to skin at the scar site, pushing the larynx to left [Fig $1 \& 2]$. There were no enlarged neck nodes. MRI showed a well defined lobulated T1 hypo intense, T2 hyper intense right submandibular lesion displacing the laryngeal framework to left. Great vessels were normal. Wide local excision was planned.

Intra operatively the lesion was found infiltrating both bellies of ipsilateral digastric muscle and hypoglossal nerve, which were resected along with the lesion. Perichondrium of thyroid cartilage was shaved off for clearance [Fig 3]. Primary wound closure was achieved after excision of the involved skin.

Cut section of specimen showed variegated surface, predominantly circumscribed glistening white lesion with hemorrhagic areas [Fig 4]. Microscopic examination revealed lobules of neoplastic chondrocytes with pleomorphic nuclei in a background of scanty amorphous chondroid matrix with infiltration into subdermal tissue and muscles. Lymph vascular invasion was present. No glandular elements were made out. There were no areas morphologically consistent with pleomorphic adenoma - histological picture was suggestive of chondrosarcoma [Fig 5, 6]. Immuno histochemical analysis revealed EMA positivity in $10-20 \%$ of tumor cells [Fig 7 - orange staining] and diffuse positivity for S100 [Fig 8], confirming carcinosarcoma.

These histological and immuno histochemical findings were consistent with malignant mixed tumor with mesenchymal chondrosarcomatous component dominating and almost entirely replacing the epithelial component.

In view of close margins, patient received adjuvant radiotherapy.

\section{Discussion}

The spectrum of malignancy in mixed tumors as already stated comprises three distinct entities. Carcinoma ex pleomorphic adenoma, Carcinosarcoma (true malignant mixed tumor) and benign metastasizing 
pleomorphic adenoma [1]. While the former two are frankly malignant, the existence of later is being questioned but recognized in WHO classification and is described as having a 40-50\% mortality rate [3]. Categorization of these is important for treatment planning and determination of the prognosis.

Carcinoma ex pleomorphic adenoma, by definition, is composed of a mixture of pleomorphic adenoma and carcinoma on microscopic examination. Though carcinomatous component replacing the benign counterpart has been reported, presence of sarcomatous and carcinomatous component rules out this entity.

Carcinosarcoma of salivary gland is an extremely rare aggressive neoplasm, first described by Kirklin et al in 1951 in parotid [4]. The term true malignant mixed tumor (carcinosarcoma) was first described by King et al. Due to its rarity, the clonality and tumurogeneis of these tumors have long been debated. Two proposed theories are, 1. 'Convergence theory' - suggests the existence of multiclonal tumor from which two or more mesenchymal and epithelial stem cells arise. 2. 'Divergence hypothesis' - claims monoclonality in which a totipotent stem cell under goes divergent differentiation [5, 6]. Loss of heterozygosity ( $\mathrm{LOH})$ analysis suggests a monoclonal origin and the myoepithelial cell is the most likely candidate for the common stem cell [7]. AlvaresCanas and Rodilla proposed an origin from the ductal luminal epithelial cells, but still primitive pleuripotent cells have been thought to be the cell of origin by other authors $[8,9]$

Most of the reported cases were in parotid with less than 15 cases reported from submandibular salivary gland. There is no gender predominance. The mean age at presentation is 58 years [14-87 years]. Many cases are seen to arise in a pre-existing benign mixed tumor with characteristic recurrent lesions with rapid growth and adjacent nerve, muscle and vessel infiltration [1]. The modes of metastases are both hematogenous and lymphatic. Hematogenous metastases are more common to the lung, bone, and liver. Cervical and hilar lymph node metastases are also common $[10,11]$.

Carcinosarcoma behave clinically aggressive with tendency to recur and metastasize. Two third of patients recur and about a half metastasize. According to the reports of Tortoledo et al [12], the 5-year survival rate with carcinosarcoma is 0\%. Another report of Gnepp and Wening [13] shows that the average survival is 29.3 months, and $23 \%$ are free of tumors for periods ranging from 5 months to 19 years. No definite association between gross dimensions of the tumor and incidences of recurrence or metastases could be made [12].

Cytological examination has limited accuracy due to tumor heterogeneity and diversity. Usually the diagnosis is confirmed by post operative histopathology, sometimes requiring immuno histochemical analysis. In most cases, the sarcomatous component dominates over the carcinomatous component. Metastatic and recurrent tumors usually manifest both the elements $[14,15]$.The epithelial component is usually a poorly differentiated adenocarcnioma, an undifferentiated carcinoma or a squamous cell carcinoma and the common types of mesenchymal components are chondrosarcoma, fibrosacroma, leiomyosarcoma, osteosarcoma, and rarely liposarcoma, in the order of decreasing frequency. Cytologic atypia, including cellular and nuclear pleomorphism, hyperchromatism, mitotic figures and invasive growth, distinguish carcinosarcomas from benign mixed tumor.

In our case, histology was not typically biphasic, rather revealing predominant chondrosarcomatous component with no carcinomatous component. Hence IHC was sought. The IHC panel varies depending upon the biphasic component observed. The carcinomatous components are usually cytokeratin and EMA positive, however very poorly differentiated tumors may show only weak or focal staining. The sarcomatous components are vimentin positive. S100 is used to detect chondromatous elements and actin for muscular component [16]. Our patient had EMA positivity in $10-20 \%$ of tumor cells and diffuse positivity for S100, confirming carcinosarcoma with chondrosarcomatous mesenchymal component.

Clinical, histological, immuno histochemical studies and their relationship to pleomorphic adenomas imply that carcinosarcoma and pleomorphic adenoma of the salivary gland may share a common precursor cell with myoepithelial cell being a major component in their development [17, 18]. On the other hand, it has been shown that some carcinosarcomas arise de novo [17, 19, 20]. It has also been demonstrated that certain carcinosarcomas do not show evidence of myoepithelial cell origin or differentiation and that they may arise independently of pleomorphic adenoma.

The differential diagnosis of carcinosarcoma includes the sarcomatoid variant of salivary duct carcinoma and Synovial sarcoma. The former have epithelial component bearing a striking resemblance to ductal carcinoma of the breast. The immuno histochemical reactivity for cytokeratin in the sarcomatous regions can be helpful, but not always, for making the diagnosis of sarcomatoid salivary duct carcinoma [21]. Synovial sarcoma will show immuno histochemical profiles similar to carcinosarcoma. Hence the histological features of tightly interlacing fascicles of uniform spindle cells and gland-like structures differentiate them from carcinosarcoma which are pleomorphic, with less integrated carcinomatous and sarcomatous tissues [1]. Collision tumors or metastatic disease should always be excluded before making a diagnosis of malignant mixed tumor [22]. 
The prognosis is unfavourable with studies reporting 56\% 5 year survival. Due to its rarity, there are no trials proving survival benefit with addition of adjuvant therapy. In view close margin our patient had received adjuvant radiation therapy.

\section{Conclusion}

In summary, we report a case of true malignant mixed tumor occurring in a background of recurrent submandibular neoplasm. Though the histology was dominated by mesenchymal component we could demonstrate the epithelial element by immunohistochemistry thereby establishing the biphasic nature of the disease.



Fig 1

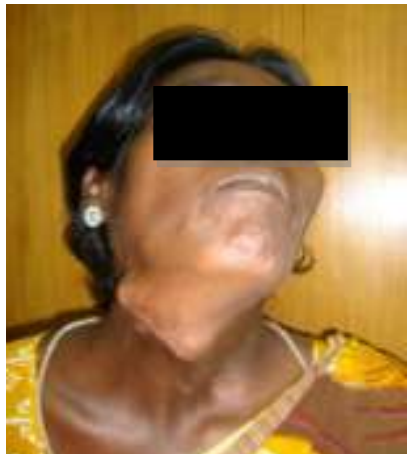

Fig 2

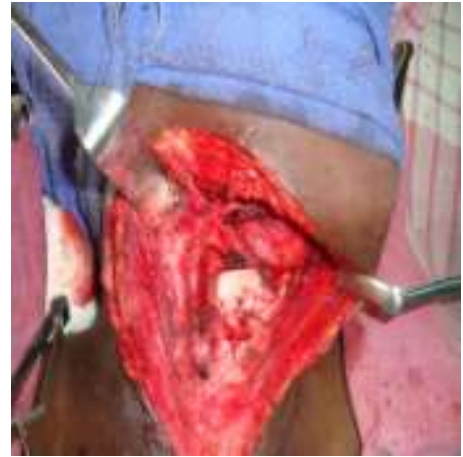

Fig 3

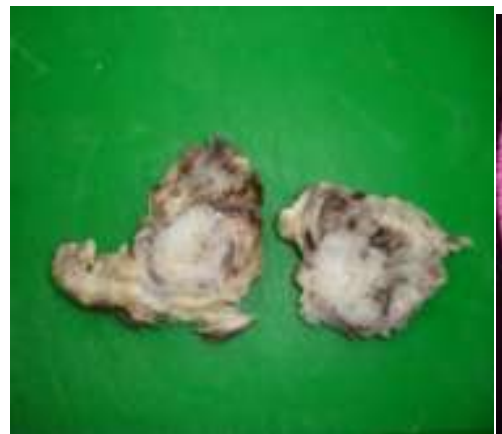

Fig 4

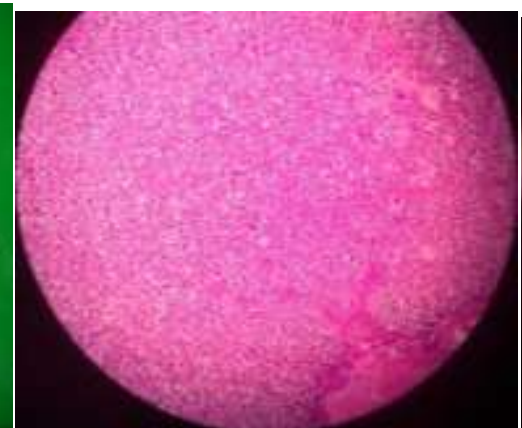

Fig 5

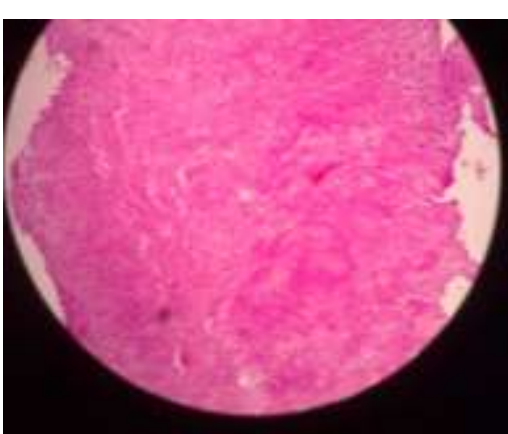

Fig 6

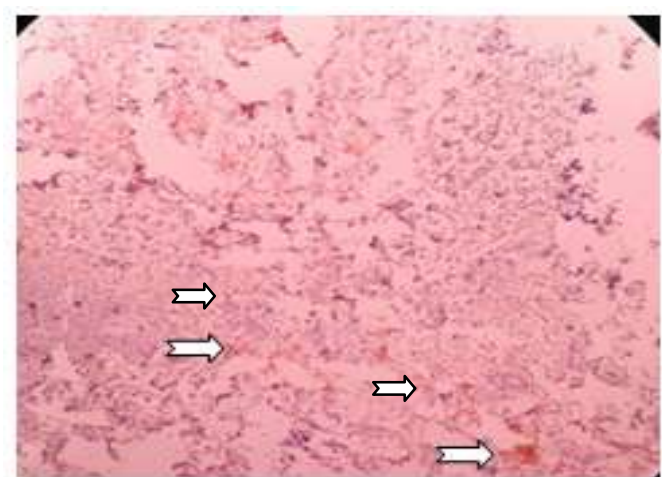

Fig 7



Fig 8

\section{References}

[1]. Gnepp DR. Malignant mixed tumors of the salivary glands: a review. Pathol Ann 1993;28:279-328

[2]. Hun-Soo Kim et al, Carcinosarcoma (True Malignant Mixed Tumor) of the Parotid Gland - A report of a Case with Small Cell Carcinoma as the Carcinoma Component The Korean Journal of Pathology 2008; 42: 175-80

[3]. Barnes L, Eveson J, Reichart PA, et al. Lymphoepithelial carcinoma. In: Kleihues P, Sobin LHeds). World Health Organization Classification of Tumors: Pathology \& Genetics of Head and Neck Tumors. IARC Press: Lyon, 2005, p18.

[4]. Kirklin JW, McDonald JR, Harrington SW, New GB. Parotid tumors: histopathology,

clinical behavior, and end results. Surg Gynecol Obstet. 1951;92:721- 733.

[5]. Thompson L, Chang B, Barsky SH., et al. Monoclonal origins of malignant mixed tumors (carcinosarcomas). Evidence for a divergent histogenesis. Am J Surg Pathol 1996; 20(3): 277-85. 
[6]. Vekony H, Leemans CR, Ylstra B, et al. Salivary gland carcinoma: oligonucleotide array CGH reveals similar genomic profiles in epithelial and mesenchymal components. Oral Oncol 2009; 45(3):259-65.

[7]. Gotte K, Riedel F, Coy JF, Spahn V, Hormann K. Salivary gland carcinosarcoma: immunohistochemical, molecular genetic and electron microscopic findings. Oral Oncol 2000; 36: 360-4.

[8]. Alvarez-Canas C, Rodilla IG, et al. True malignant mixed tumor (carcinosarcoma) of the parotid gland. Report of a case with immunohistochemical study. Oral Surg Oral Med Oral Pathol Oral Radiol Endod 1996;81(4):454-8.

[9]. Bleiweiss IJ, Huvos AG, Lara J, et al. Carcinosarcoma of the submandibular salivary gland. Immunohistochemical findings. Cancer 1992;69(8):2031-5.

[10]. Stephen J, Batsakis JG, Luna MA, Heyden U, Byers RM. True malignant mixed tumors carcinosarcoma) of the salivary glands. Oral Surg Oral Med Oral Pathol 1986; 61: 597-602.

[11]. Ellis GL, Auclair PL. Tumors of the salivary glands. 3rd ed. Washington (DC): Armed Forces Institute of Pathology 1996; $238-45$.

[12]. Tortoledo ME, Luna MA, Batsakis JG. Carcinomas ex pleomorphic adenoma and malignant mixed tumors. Arch Otolaryngol 1984;110:172-176

[13]. Gnepp DR, Wening BM. Malignant mixed tumors. In: Ellis GL, Auclair PL, Gnepp DR, eds. Surgical Pathology of the Salivary Glands. Philadelphia, Pa: WB Saunders Co, 1991:357-363

[14]. Dardick I, Hardie J, Thomas MJ, Van Nostrand AW. Ultrastructural differentiation in malignant mixed tumors of salivary gland. Head Neck 1989;11:5-21.

[15]. Di Palma S, Guzzo M. Malignant myoepithelioma of salivary glands: Clinicopathologic features of 10 cases. Virchows Arch A Pathol Anat Histopathol 1993;423:389-96.

[16]. Anamaria Mag et al, True Malignant Mixed Tumor (Carcinosarcoma) Of The Parotid Gland - Case Report, TMJ 2010, Vol. 60 , No. 4

[17]. Bleiweiss IJ, Huvos AG, Lara J, Strong EW. Carcinosarcoma of the submandibular salivary gland: immunohistochemical findings. Cancer. 1992;69:2031- 2035

[18]. Stephen J, Batsakis JG, Luna MA, von der Heyden U, Byers RM. True malignant mixed tumors (carcinosarcoma) of salivary glands. Oral Surg Oral Med Oral Pathol. 1986;61:597-602

[19]. Grenko RT, Tytor M, Boeryd B. Giant-cell tumor of the salivary gland with associated carcinosarcoma. Histopathology. 1993;23:594-595

[20]. Latkovich P, Johnson RL. Carcinosarcoma of the parotid gland: report of a case with cytohistologic and immunohistological findings. Arch Pathol Lab Med. 1998; 122:743-746.

[21]. Nagao T, Gaffey TA, Serizawa H, et al. Sarcomatoid variant of salivary duct carcinoma: clinicopathologic and immunohistochemical study of eight cases with review of the literature. Am J Clin Pathol 2004; 122: 222-31.

[22]. Latkovich P, Johnson RL. Carcinosarcoma of the parotid gland: report of a case with cytohistologic and immunohistochemical findings. Arch Pathol Lab Med 1998; 122: 743-6. 